\title{
A prospective study of obstetric and gynaecological emergencies in a tertiary care hospital
}

\author{
Shahna Anishbhai Jindani, Asha Bhagwatibhai Sailor*, Dipti A. Modi, Somika Kaul, \\ Bijal D. Rami
}

Department of Obstetrics and Gynecology, Government Medical College, Baroda, Gujarat, India

Received: 20 February 2020

Revised: 18 March 2020

Accepted: 27 March 2020

\section{*Correspondence:}

Dr. Asha Bhagwatibhai Sailor,

E-mail: ashasailor63@gmail.com

Copyright: (c) the author(s), publisher and licensee Medip Academy. This is an open-access article distributed under the terms of the Creative Commons Attribution Non-Commercial License, which permits unrestricted non-commercial use, distribution, and reproduction in any medium, provided the original work is properly cited.

\section{ABSTRACT}

Background: The management of obstetrics and gynaecological emergency is directed at the preservation of life, health, sexual function and the perpetuation of fertility. The main aim of the study was to access the burden of surgical emergency and to study the course of management at a tertiary care hospital.

Methods: This prospective study was carried out in the department of obstetrics and gynaecology, S. S. G. Hospital, Baroda for a period from January to December 2018.

Results: A total of 73 patients presented to our emergency room who required urgent surgical intervention. All patients were resuscitated and surgery was done at earliest possible time. The age of patient ranged from 18 to 45 years. About $75.8 \%$ of female presented with the complaint of acute abdomen, followed by $32.9 \%$ with bleeding per vaginum. $16.4 \%$ had vomiting, $6.8 \%$ with fever and $4.1 \%$ with mass per abdomen. In majority of cases a diagnosis of ruptured ectopic pregnancy (34 patients) was made, followed by PPH in 14 patients and 12 cases of rupture uterus. Four cases of torsion of ovarian mass and 3 cases of septic peritonitis were operated. The most common surgery performed was salpingectomy followed by subtotal obstetric hysterectomy. A mortality rate of $8.2 \%$ was noted.

Conclusions: This study emphasized the great role of timely surgical intervention as lifesaving procedures. Skilled clinicians and immediate intervention in a tertiary care is the main-stay of the emergency case management and are indispensable for decreasing mortality and morbidity.

Keywords: Ectopic pregnancy, Hysterectomy, Postpartum haemorrhage, Rupture uterus

\section{INTRODUCTION}

The management of women presenting to the emergency department of obstetrics or gynaecology has the main aim to prevent maternal mortality and morbidity and thereby optimize women health. Most of the patients present with acute abdomen, abnormal vaginal bleeding or a combination of both. The developments of ultrasonographic imaging, serial biochemical pregnancy tests and minimal invasive surgery have given opportunity for early diagnosis and conservative treatment. ${ }^{1}$ The common causes of a female presenting to the emergency department of obstetrics and gynaecology are as follows.

Ectopic pregnancy is a life-threatening gynaecological emergency and a leading cause of maternal mortality in the early half of pregnancy. ${ }^{2,3}$ The incidence of ectopic pregnancy is increasing worldwide. ${ }^{4}$ Delay in the diagnosis can be catastrophic due to associated haemorrhage. Postpartum haemorrhage is one of the leading causes of maternal mortality and morbidity and represents the most challenging complication that an obstetrician will face. ${ }^{5}$ Severe postpartum haemorrhage 
was reported to occur in 6.7/1000 deliveries world-wide. ${ }^{6}$ Rupture uterus is a catastrophic complication which occurs most common in women attempting a vaginal birth after a prior caesarean section but rupture of a nulliparous unscarred uterus, though rare is also possible. In women who undergo atrial of labour after one low transverse caesarean section, the incidence of rupture uterus is estimated to be less than $1 \%$ and the rate of successful vaginal delivery varies from $60 \%-80 \%{ }^{7}$ Tumors of the ovary are common in women, with about $80 \%$ being benign and occurring in the reproductive age group. ${ }^{8}$ Generally, ovarian cysts that are painful may be as a result of torsion, haemorrhage and rupture. Torsion of the ovarian cyst commonly present with severe acute lower abdominal pain that is often associated with nausea and vomiting. Gynaecologic disorders presenting with acute abdomino-pelvic pain may be benign and selflimiting or may negatively affect fertility capacity if not treated. The incidence is calculated as $5 \%$ of emergency department admissions. The delay in diagnosis and treatment may lead to worse outcomes. ${ }^{9,10}$ This study was carried out with an objective of evaluating the gravity of emergencies and to highlight various obstetrical and gynaecological problems presenting as acute surgical emergencies and their management at the institute.

\section{METHODS}

This prospective study was carried out in the department of obstetrics and gynecology, S. S. G. Hospital Baroda from January 2018 till December 2018. A total number of 73 patients were followed who presented to emergency room of the department and required urgent surgical intervention during the study period. Immediate resuscitation followed by detailed history taking, physical examinations, laboratory investigations including ultrasonography for diagnosis and appropriate management were done as per the existing protocol for all patients. Data was collected using semi-structured questionnaire, treatment records and intraoperative findings after taking consent from the patient's closest visitor.

\section{Inclusion criteria}

- Booked or unbooked cases

- Patients who presented to the obstetrics and gynaecology emergency and required an urgent surgical intervention.

\section{Exclusion criteria}

- Patients requiring conservative management

- Patients requiring a caesarean delivery.

\section{Statistical analysis}

The results were presented as numbers and percentages and were analysed using medcalc software. The analysed data was compared with different studies and discussed.

\section{RESULTS}

Out of a total of 73 patients in the study, the highest number of patients $48(65.8 \%)$ was seen in the age group of 25 to 35 years, which was followed by $14(19.2 \%)$ in the age group of more than 35 years category and 11 (15\%) of the patients were present in the less than 25 years category.

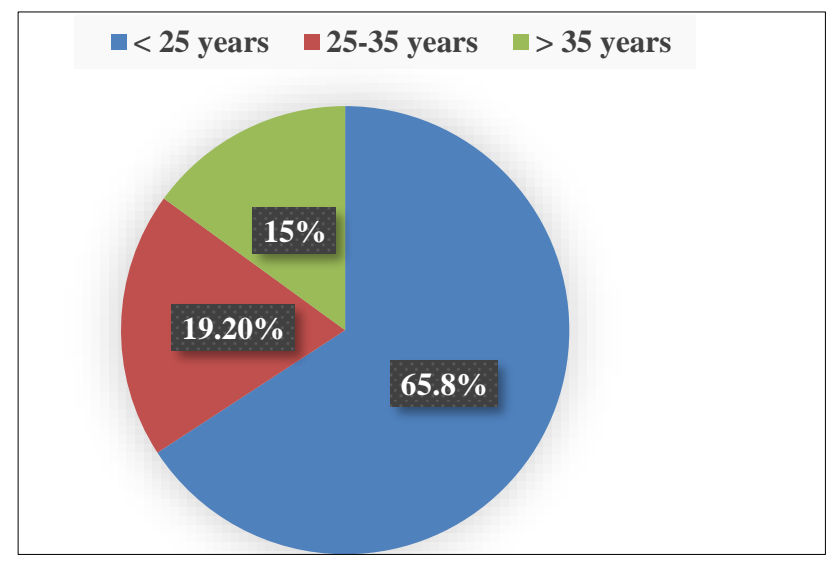

Figure 1: Distribution of patients according to age.

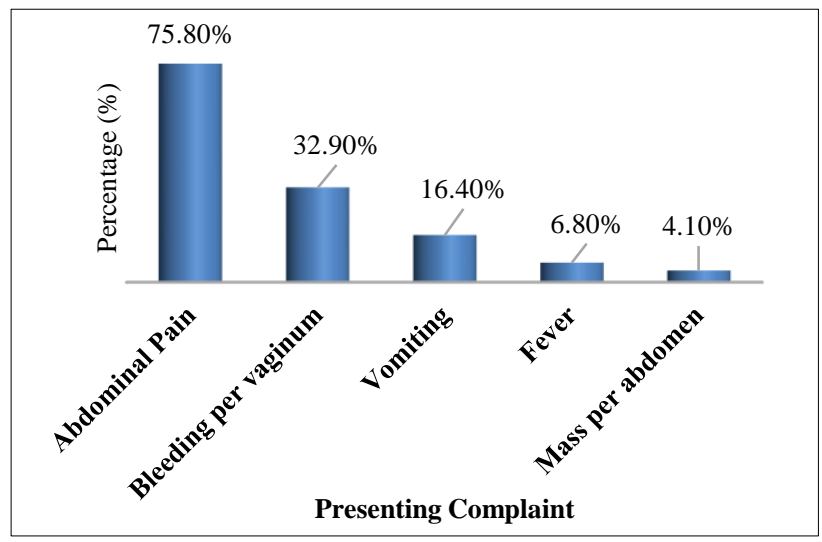

Figure 2: Distribution of patients according to presenting complaints.

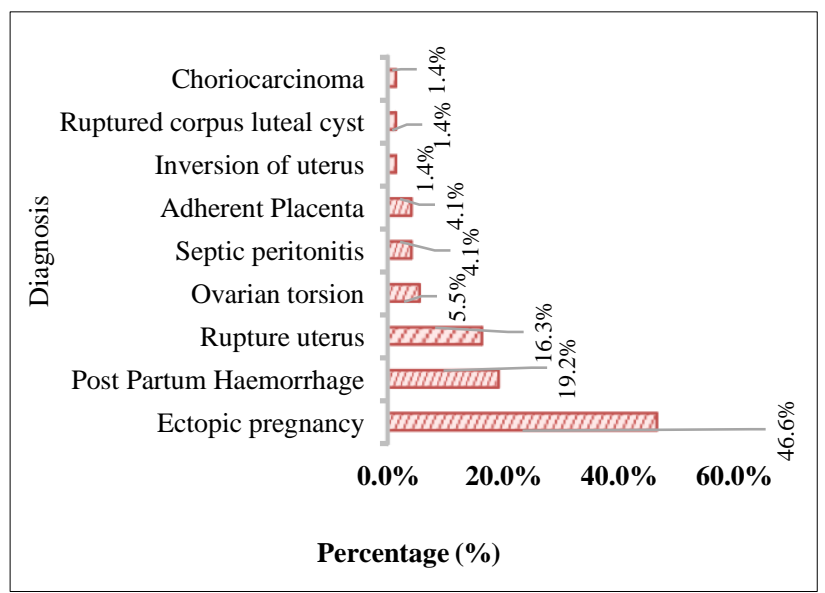

Figure 3: Distribution according to the diagnosis. 
The most common presenting complaint was pain abdomen, seen in $55(75.8 \%)$ patients followed by bleeding per vagina among $24(32.9 \%)$ patients and vomiting in $12(16.4 \%)$ patients. Fever was the presenting complain in $5(6.8 \%)$ patients and $3(4.1 \%)$ patients presented with mass per abdomen.

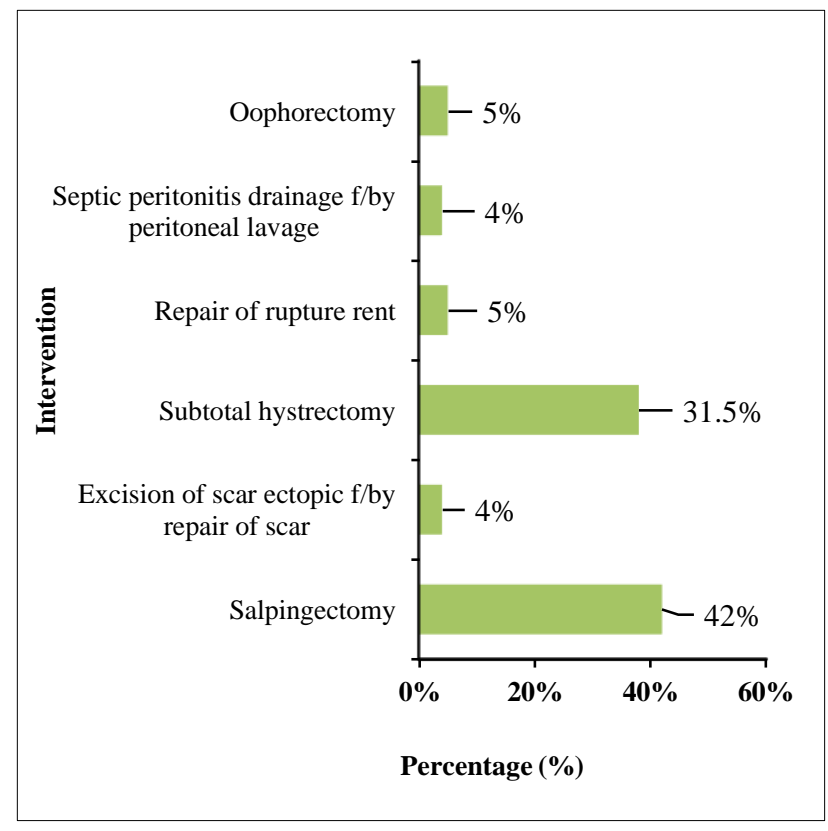

Figure 4: Intervention required.

The most common diagnosis made was ruptured ectopic pregnancy, in 34 patients $(46.6 \%)$. This was followed by postpartum haemorrhage in 14 patients (19.2\%) and rupture uterus in 12 cases $(16.3 \%)$. Ovarian torsion was seen in 4 cases $(5.5 \%)$. Both septic peritonitis and adherent placenta was seen in $(4.1 \%) 3$ patients each.

A total of $31(42.5 \%)$ patients underwent salpingectomy and $23(31.5 \%)$ patients required subtotal obstetric hysterectomy. In $3(4.1 \%)$ patients, scar ectopic was excised followed by (f/by) scar repair. In $4(5.5 \%)$ patients, repair of rupture uterus rent was done. $3(4.1 \%)$ patients underwent peritoneal lavage for septic peritonitis. Oophrectomy was done in $4(5.5 \%)$ patients for ovarian torsion.

\section{DISCUSSION}

A wide variety of patients with surgical emergencies in obstetrics and gynecology are encountered in emergency room starting from young girls to older women and nulliparous to multiparous females. In the present study we analyzed 73 patients on the basis of patient age at the time of presentation to the emergency, presenting symptom, diagnosis made and surgical intervention required.

In the present study $65.8 \%$ patients were in the age group of 25 to 35 years. In a similar study done by Hanoon P et al $42.7 \%$ patients were in the age group of 25 to 34 years. $^{11}$

Maximum number of patients presented with the complain of pain abdomen (75.8\%). while as $32.9 \%$ patients presented with bleeding per vaginum, $16.4 \%$ had vomiting and $6.85 \%$ had fever. Also, $58.9 \%$ patients had haemoperitoneum. In the study done by Hanoon $\mathrm{P}$ et al similar results were obtained. ${ }^{11}$ In that study, $75.8 \%$ patients presented with pain abdomen, $21.0 \%$ presented with bleeding per vaginum and $10.85 \%$ had vomiting. In the study done by Barat $\mathrm{S}$ et al it was seen that $55.1 \%$ of patients complained of having pain lasting more than 24 hours and abnormal vaginal bleeding was present in $27.1 \%$ of patients. ${ }^{12}$

A total of 34 patients $(46.6 \%)$ of ruptured ectopic pregnancies were seen in the present study. $19.2 \%$ of patients were diagnosed as having postpartum haemorrhage and $16.3 \%$ were having rupture uterus. In a similar study done in Nepal, $61.1 \%$ patients were diagnosed with ectopic pregnancy followed by ovarian mass in $7.6 \%$. Postpartum haemorrhage and rupture uterus were seen in $3.2 \%$ and $5.7 \%$ patients respectively. ${ }^{11}$ In a study done by Awori $\mathrm{MN}$ et al, in female patients presenting with abdominal pain, the incidence of ectopic pregnancy was $65.3 \% .^{13}$

In the present study $42.5 \%$ patients required salpingectomy and $31.5 \%$ underwent subtotal obstetric hysterectomy. Similar results were obtained in a study by Hanoon $\mathrm{P}$ et al in which $47.8 \%$ patients required salpingectomy and $37.0 \%$ patients had to undergo subtotal abdominal hysterectomy or total abdominal hysterectomy with bilateral salpingo-ophrectomy was done. ${ }^{11}$ In the present study, mortality was noted in $8.2 \%$ cases.

\section{CONCLUSION}

This study revealed the common obstetrical and gynaecological emergencies encountered in a tertiary care centre. At the same time, it was observed that mortality and morbidity resulting from such conditions can be avoided with minimal precautions, proper counselling, quick diagnosis, prompt and aggressive management, multidisciplinary approach and referral at appropriate time.

This study highlighted that there is a great role of timely surgical intervention as a life- saving procedures in a tertiary care hospital. Skilled clinicians and immediate intervention in a tertiary care centre are the main backbone of the emergency case management and saving lives.

Funding: No funding sources

Conflict of interest: None declared

Ethical approval: Not required 


\section{REFERENCES}

1. Ramphal SR, Moodley J. Emergency gynaecology. Best Pract Res Clin Obstet Gynaecol. 2006;20:72950.

2. Olarewaju RS, Ujah IOA, Otubu JAM. Trends in ectopic pregnancy in the Jos University Teaching Hospital, Jos, Nieria. Nig J Med. 1994;26:57-60.

3. Grimes DA. The morbidity and mortality of pregnancy. Still risky business. Am J Obstet Gynecol. 1994;170:1489-94.

4. Pisarka MD, Carson SA, Buster JE. Ectopic pregnancy. Lancet. 1998;351:115-20.

5. Kashani E, Azarhoush R. Peripartum hysterectomy for primary postpartum hemorrhage: 10 years evaluation. J Expo Biol. 2012;(1):32-6.

6. Ferreira Carvalho J, Cubal A, Torres S, Costa F, Carmo OD. Emergency peripartum hysterectomy: a 10-year review. ISRN Emerg Med. 2012:2012.

7. Smith JG, Mertz HL, Merrill DC. Identifying risk factors for uterine rupture. Clin Perinatol. 2008;35:85-99.

8. Cotran RS, Kumar V, Collins T. Ovarian tumours. Robbins pathological basis of disease. $6^{\text {th }}$ edition; 1999:1065-1079

9. Kamin RA, Nowicki TA, Courtney DS, Powers RD. Pearls and pitfalls in the emergency department evaluation of abdominal pain. Emerg Med Clin North Am. 2003;21:61-72.

10. Jermy K, Bourne T. The role of ultrasound in the management of acute gynecological abdomen. Rev Gynaecol Pract. 2004;4:224-9.

11. Pokharel HP, Dahal P, Rai R, Budhathoki SS. Surgical emergencies in obstetrics and gynaecology in atertiaryhospital. $\mathrm{J}$ Nepal Med Assoc. 2013;52(189):213-6.

12. Barat S, Bouzari Z, Nilbakhsh N, Rad MN. Acute obstetric and gynecologic emergencies among pregnant women in a tertiary care hospital, Iran. Caspian J Reprod Med. 2015;1(2):6-9.

13. Awori MN, Jani G. Surgical implications of abdominal pain in patients presenting to the Kenyatta National Hospital casualty department with abdominal pain. East Afr Med J. 2005;82:307-10.

Cite this article as: Jindani SA, Sailor AB, Modi DA, Kaul S, Rami BD. A prospective study of obstetric and gynaecological emergencies in a tertiary care hospital. Int J Reprod Contracept Obstet Gynecol 2020;9:1992-5. 